\title{
The common solutions of the split feasibility problems and fixed point problems
}

Abdelouahed Hamdi', Yeong-Cheng Liou ${ }^{2,3}$, Yonghong Yao ${ }^{4 *}$ and Chongyang Luo ${ }^{4}$

\section{"Correspondence:}

yaoyonghong@aliyun.com

${ }^{4}$ Department of Mathematics,

Tianjin Polytechnic University,

Tianjin, 300387, China

Full list of author information is

available at the end of the article

\begin{abstract}
An extraordinary split problem, which can be regarded as a superimposition of the split feasibility problem and the split fixed point problem, is considered. A superimposed algorithm is presented. The analysis technique of the suggested algorithm and the corresponding convergence results are demonstrated.
\end{abstract}

MSC: 47J25; 47H09; 65J15; 90C25

Keywords: split feasibility problem; split fixed point problem; iterative algorithm; quasi-pseudo-contraction; strong convergence

\section{Introduction}

\subsection{Background}

The split feasibility problem (SFP) is formulated as finding $u^{\ddagger}$ such that

$$
u^{\ddagger} \in \mathcal{C} \quad \text { and } \quad \mathcal{A} u^{\ddagger} \in \mathcal{Q} \quad\left(\text { or } u^{\ddagger} \in \mathcal{C} \cap \mathcal{A}^{-1} \mathcal{Q} \text { when } \mathcal{A}^{-1}\right. \text { exists), }
$$

where $\mathcal{C}(\neq \emptyset)$ and $\mathcal{Q}(\neq \emptyset)$ are closed convex subsets of real Hilbert spaces $\mathcal{H}_{1}$ and $\mathcal{H}_{2}$, respectively, and $\mathcal{A}$ is a bounded linear operator from $\mathcal{H}_{1}$ to $\mathcal{H}_{2}$. The mathematical model of the SFP was refined from phase retrievals and the medical image reconstruction by Censor and Elfving [1] in 1994. One effective approach to solve the SFP is algorithmic iteration. There are several effective iterations which are listed as follows.

Existing iterations for the SFP 1 . Simultaneous multiprojections (Censor and Elfving [1]):

$$
x_{k+1}=\mathcal{A}^{-1} \operatorname{proj}_{\mathcal{Q}}\left(\operatorname{proj}_{\mathcal{A}(\mathcal{C})}\left(\mathcal{A} x_{k}\right)\right), \quad k \in \mathbb{N},
$$

where $\mathcal{C} \subset \mathbb{R}^{n}$ and $\mathcal{Q} \subset \mathbb{R}^{n}$ are closed convex sets, and $\mathcal{A}$ is an $n \times n$ matrix.

2. Gradient projections (CQ iteration) [2-6]:

$$
x_{k+1}=\operatorname{proj}_{\mathcal{C}}\left(x_{k}-\frac{\varpi}{\|\mathcal{A}\|^{2}} \mathcal{A}^{T}\left(\mathcal{I}-\operatorname{proj}_{\mathcal{Q}}\right) \mathcal{A} x_{k}\right), \quad k \in \mathbb{N},
$$

where $\varpi$ is a constant and $\mathcal{A}^{T}$ denotes the transposition of $\mathcal{A}$.

(c) 2015 Hamdi et al. This article is distributed under the terms of the Creative Commons Attribution 4.0 International License (http://creativecommons.org/licenses/by/4.0/), which permits unrestricted use, distribution, and reproduction in any medium, provided you give appropriate credit to the original author(s) and the source, provide a link to the Creative Commons license, and indicate if changes were made. 
3. Averaged CQ iteration [2, 7]:

$$
x_{k+1}=\left(1-\alpha_{k}\right) x_{k}+\alpha_{k} \operatorname{proj}_{\mathcal{C}}\left(x_{k}-\frac{\varpi}{\|\mathcal{A}\|^{2}} \mathcal{A}^{*}\left(\mathcal{I}-\operatorname{proj}_{\mathcal{Q}}\right) \mathcal{A} x_{k}\right), \quad k \in \mathbb{N},
$$

where $\left.\alpha_{k} \in\right] 0,1\left[\right.$, $\varpi$ is a constant and $\mathcal{A}^{*}$ is the adjoint of $\mathcal{A}$.

4. Relaxed CQ iteration $[3,8,9]:$ Let $f: \mathcal{H}_{1} \rightarrow \mathbb{R}$ and $g: \mathcal{H}_{2} \rightarrow \mathbb{R}$ be two convex functions. Define two level sets and the related subdifferentials

$$
\begin{aligned}
& \mathcal{C}:=\left\{x \in \mathcal{H}_{1} \mid f(x) \leq 0\right\} \quad \text { and } \quad \mathcal{Q}:=\left\{y \in \mathcal{H}_{2} \mid g(y) \leq 0\right\} \\
& \partial f(x)=\left\{z \in \mathcal{H}_{1} \mid f(u) \geq f(x)+\langle u-x, z\rangle, u \in \mathcal{H}_{1}\right\}, \quad \forall x \in \mathcal{C}
\end{aligned}
$$

and

$$
\partial g(x)=\left\{w \in \mathcal{H}_{2} \mid g(v) \geq g(y)+\langle v-y, w\rangle, v \in \mathcal{H}_{2}\right\}, \quad \forall y \in \mathcal{Q} .
$$

Define the relaxed CQ iteration as follows:

$$
x_{k+1}=\operatorname{proj}_{\mathcal{C}_{k}}\left(x_{k}-\frac{\varpi}{\|\mathcal{A}\|^{2}} \mathcal{A}^{T}\left(\mathcal{I}-\operatorname{proj}_{\mathcal{Q}_{k}}\right) \mathcal{A} x_{k}\right), \quad k \in \mathbb{N},
$$

where

$$
\mathcal{C}_{k}=\left\{x \in \mathcal{H}_{1} \mid f\left(x_{k}\right)+\left\langle\xi_{k}, x-x_{k}\right\rangle \leq 0\right\},
$$

where $\xi_{k} \in \partial f\left(x_{k}\right)$, and

$$
\mathcal{Q}_{k}=\left\{y \in \mathcal{H}_{2} \mid g\left(\mathcal{A} x_{k}\right)+\left\langle\eta_{k}, y-\mathcal{A} x_{k}\right\rangle \leq 0\right\},
$$

where $\eta_{k} \in \partial g\left(\mathcal{A} x_{k}\right)$.

5. Regularized iteration $[2,10]$ :

$$
x_{k+1}=\operatorname{proj}_{\mathcal{C}}\left(\left(1-\alpha_{k} \varpi_{k}\right) x_{k}-\varpi_{k} \mathcal{A}^{*}\left(\mathcal{I}-\operatorname{proj}_{\mathcal{Q}}\right) \mathcal{A} x_{k}\right), \quad k \in \mathbb{N},
$$

where $\left.\left\{\alpha_{k}\right\} \subset\right] 0,1\left[\right.$ and $\left.\left\{\varpi_{k}\right\} \in\right] 0, \frac{\alpha_{k}}{\|\mathcal{A}\|^{2}+\alpha_{k}}[$.

6. Self-adaptive iteration [11-13]:

$$
x_{k+1}=\operatorname{proj}_{\mathcal{C}}\left(x_{k}-\varpi_{k} \mathcal{A}^{*}\left(\mathcal{I}-\operatorname{proj}_{\mathcal{Q}}\right) \mathcal{A} x_{k}\right), \quad k \in \mathbb{N},
$$

where the step-size $\varpi_{k}=\frac{\tau_{k}\left\|\left(\mathcal{I}-\operatorname{proj}_{\mathcal{Q}}\right) \mathcal{A} x_{k}\right\|^{2}}{2\left\|\mathcal{A} *\left(\mathcal{I}-\operatorname{proj}_{\mathcal{Q}}\right) \mathcal{A} x_{k}\right\|^{2}}$ in which $\left.\tau_{k} \in\right] 0,2[$.

7. Halpern-type iteration [2]:

$$
x_{k+1}=\alpha_{k} u+\left(1-\alpha_{k}\right) \operatorname{proj}_{\mathcal{C}}\left(x_{k}-\varpi_{k} \mathcal{A}^{*}\left(\mathcal{I}-\operatorname{proj}_{\mathcal{Q}}\right) \mathcal{A} x_{k}\right), \quad k \in \mathbb{N},
$$

where $u \in \mathcal{C}$ is a fixed point, $\left.\left\{\alpha_{k}\right\} \subset\right] 0,1\left[\right.$ and $\varpi_{k}=\frac{\tau_{k}\left\|\left(\mathcal{I}-\operatorname{proj}_{\mathcal{Q}}\right) \mathcal{A} x_{k}\right\|^{2}}{2\left\|\mathcal{A}^{*}\left(\mathcal{I}-\operatorname{proj}_{\mathcal{Q}}\right) \mathcal{A} x_{k}\right\|^{2}}$ in which $\left.\tau_{k} \in\right] 0,2[$. 
The (two-set) split common fixed point problem (SCFP) can be formulated as finding $u^{\dagger}$ such that

$$
u^{\dagger} \in \operatorname{Fix}(\mathcal{T}) \text { and } \mathcal{A} u^{\dagger} \in \operatorname{Fix}(\mathcal{S})
$$

where $\operatorname{Fix}(\mathcal{T})$ and $\operatorname{Fix}(\mathcal{S})$ stand for the fixed point sets of the operators $\mathcal{T}: \mathcal{H}_{1} \rightarrow \mathcal{H}_{1}$ and $\mathcal{S}: \mathcal{H}_{2} \rightarrow \mathcal{H}_{2}$.

The SCFP is a natural extension of the SFP and of the convex feasibility problem. The SCFP was firstly considered by Censor and Segal in [14] where $\mathcal{S}$ and $\mathcal{T}$ are directed operators which include the orthogonal projections and the sub-gradient projectors.

Existing iterations for the SCFP 1. Censor and Segal's iteration [14]:

$$
x_{k+1}=\mathcal{T}\left(x_{k}-\frac{\varpi}{\|\mathcal{A}\|^{2}} \mathcal{A}^{*}(\mathcal{I}-\mathcal{S}) \mathcal{A} x_{k}\right), \quad k \in \mathbb{N} .
$$

2. Averaged iteration $[15,16]$ :

$$
\left\{\begin{array}{l}
y_{k}=x_{k}-\frac{\omega}{\|\mathcal{A}\|^{2}} \mathcal{A}^{*}(\mathcal{I}-\mathcal{S}) \mathcal{A} x_{k} \\
x_{k+1}=\left(1-\alpha_{k}\right) y_{k}+\alpha_{k} \mathcal{T} y_{k}, \quad k \in \mathbb{N}
\end{array}\right.
$$

3. Halpern-type iteration [17]:

$$
x_{k+1}=\alpha_{k} u+\left(1-\alpha_{k}\right) \mathcal{T}\left(x_{k}-\frac{\varpi}{\|\mathcal{A}\|^{2}} \mathcal{A}^{*}(\mathcal{I}-\mathcal{S}) \mathcal{A} x_{k}\right), \quad k \in \mathbb{N} .
$$

4. Self-adaptive iteration [18]:

$$
\left\{\begin{array}{l}
y_{k}=x_{k}-\varpi_{k} \mathcal{A}^{*}(\mathcal{I}-\mathcal{S}) \mathcal{A} x_{k}, \\
x_{k+1}=(1-\lambda) y_{k}+\lambda_{k} \mathcal{T} y_{k}, \quad k \in \mathbb{N}
\end{array}\right.
$$

where the step-size $\varpi_{k}=\frac{(1-\tau)\left\|(\mathcal{I}-\mathcal{S}) \mathcal{A} x_{k}\right\|^{2}}{2\left\|\mathcal{A}^{*}(\mathcal{I}-\mathcal{S}) \mathcal{A} x_{k}\right\|^{2}}$.

5. Composite iteration [19]:

$$
\left\{\begin{array}{l}
v_{k}=x_{k}+\frac{\delta}{\|\mathcal{A}\|^{2}} \mathcal{A}^{*}\left[\left(1-\zeta_{k}\right) \mathcal{I}+\zeta_{k} \mathcal{S}\left(\left(1-\eta_{k}\right) \mathcal{I}+\eta_{k} \mathcal{S}\right)-\mathcal{I}\right] \mathcal{A} x_{k} \\
u_{k}=\alpha_{n} h\left(x_{k}\right)+\left(\mathcal{I}-\alpha_{k} \mathcal{B}\right) v_{k} \\
x_{k+1}=\left(1-\beta_{k}\right) u_{k}+\beta_{k} \mathcal{T}\left(\left(1-\gamma_{k}\right) u_{n}+\gamma_{k} \mathcal{T} u_{k}\right), \quad k \in \mathbb{N}
\end{array}\right.
$$

where $\left\{\alpha_{k}\right\}_{k \in \mathbb{N}},\left\{\beta_{k}\right\}_{k \in \mathbb{N}},\left\{\gamma_{k}\right\}_{k \in \mathbb{N}},\left\{\zeta_{k}\right\}_{k \in \mathbb{N}}$ and $\left\{\eta_{k}\right\}_{k \in \mathbb{N}}$ are five real number sequences in ]0,1[, $\delta \in] 0,1$ [ is a constant, $h: \mathcal{H}_{1} \rightarrow \mathcal{H}_{1}$ is a contraction and $\mathcal{B}: \mathcal{H}_{1} \rightarrow \mathcal{H}_{1}$ is a strong positive linear bounded operator.

\subsection{Problem statement}

The purpose of this paper is to study the following split feasibility problem and fixed point problem:

$$
\text { Find } u^{\dagger} \in \mathcal{C} \cap \operatorname{Fix}(\mathcal{T}) \text { such that } \mathcal{A} u^{\dagger} \in \mathcal{Q} \cap \operatorname{Fix}(\mathcal{S}) \text {. }
$$

It is obvious that (1.15) includes SFP (1.1) and SCFP (1.9) as special cases. 
Motivated by iterations (1.3), (1.11) and (1.14), we will construct a new iteration to approach the solution of (1.15). Strong convergence results are given in the third section.

\section{Several notions and lemmas}

Assume that $\mathcal{H}$ is a real Hilbert space. $\langle\cdot, \cdot\rangle$ and $\|\cdot\|$ stand for its inner product and norm, respectively. Let $(\emptyset \neq) \mathcal{C} \subset \mathcal{H}$ be a closed convex set.

Definition 2.1 An operator $\mathcal{P}: \mathcal{C} \rightarrow \mathcal{C}$ is said to be $\mathcal{L}$-Lipschitzian if

$$
\left\|\mathcal{P} u-\mathcal{P} u^{\dagger}\right\| \leq \mathcal{L}\left\|u-u^{\dagger}\right\|, \quad \forall u, u^{\dagger} \in \mathcal{C}
$$

for some constant $\mathcal{L}>0$.

If $\mathcal{L} \in[0,1[$, then $\mathcal{P}$ is called $\mathcal{L}$-contraction. If $\mathcal{L}=1$, then $\mathcal{P}$ is called nonexpansive.

Definition 2.2 An operator $\mathcal{P}: \mathcal{C} \rightarrow \mathcal{C}$ is said to be firmly nonexpansive if

$$
\left\|\mathcal{P} u-\mathcal{P} u^{\dagger}\right\|^{2} \leq\left\|u-u^{\dagger}\right\|^{2}-\left\|(\mathcal{I}-\mathcal{P}) u-(\mathcal{I}-\mathcal{P}) u^{\dagger}\right\|^{2}
$$

for all $u, u^{\dagger} \in \mathcal{C}$.

Definition 2.3 An operator $\mathcal{P}: \mathcal{C} \rightarrow \mathcal{C}$ is said to be pseudo-contractive if

$$
\left\langle\mathcal{P} u-\mathcal{P} u^{\dagger}, u-u^{\dagger}\right\rangle \leq\left\|u-u^{\dagger}\right\|^{2}
$$

for all $u, u^{\dagger} \in \mathcal{C}$.

Definition 2.4 An operator $\mathcal{P}: \mathcal{C} \rightarrow \mathcal{C}$ is said to be quasi-pseudo-contractive if

$$
\left\|\mathcal{P} u-u^{\dagger}\right\|^{2} \leq\left\|u-u^{\dagger}\right\|^{2}+\|\mathcal{P} u-u\|^{2}
$$

for all $u \in \mathcal{C}$ and $u^{\dagger} \in \operatorname{Fix}(\mathcal{P})$.

Definition 2.5 An operator $\mathcal{P}$ is said to be demiclosed if $\forall u_{n} \rightarrow u^{\ddagger}$ weakly and $\mathcal{P}\left(x_{n}\right) \rightarrow u$ strongly imply that $\mathcal{P}\left(u^{\ddagger}\right)=u$.

Lemma 2.6 ([20]) Let $\left\{\varrho_{n}\right\} \subset\left[0,+\infty\left[,\left\{\vartheta_{n}\right\} \subset\right] 0,1\left[\right.\right.$ and $\left\{\eta_{n}\right\}$ be three real number sequences. Suppose that $\left\{\varrho_{n}\right\},\left\{\vartheta_{n}\right\}$ and $\left\{\eta_{n}\right\}$ satisfy the following three conditions:

(i) $\varrho_{n+1} \leq\left(1-\vartheta_{n}\right) \varrho_{n}+\eta_{n} \vartheta_{n}$,

(ii) $\sum_{n=1}^{\infty} \vartheta_{n}=\infty$,

(iii) $\limsup _{n \rightarrow \infty} \eta_{n} \leq 0$ or $\sum_{n=1}^{\infty}\left|\eta_{n} \vartheta_{n}\right|<\infty$.

Then $\lim _{n \rightarrow \infty} \varrho_{n}=0$.

Lemma 2.7 ([21]) Let $\left\{\rho_{n}\right\}$ be a sequence of real numbers. Assume that there exists a subsequence $\left\{\rho_{n_{k}}\right\}$ of $\left\{\rho_{n}\right\}$ such that $\rho_{n_{k}} \leq \rho_{n_{k}+1}$ for all $k \geq 0$. For every $n \geq N_{0}$, define an integer sequence $\{\tau(n)\}$ as

$$
\tau(n)=\max \left\{i \leq n: \rho_{n_{i}}<\rho_{n_{i}+1}\right\} .
$$


Then $\tau(n) \rightarrow \infty$ as $n \rightarrow \infty$ and, for all $n \geq N_{0}$,

$$
\max \left\{\rho_{\tau(n)}, \rho_{n}\right\} \leq \rho_{\tau(n)+1}
$$

\section{Algorithms and convergence}

In this section, we first construct an iterative algorithm for solving problem (1.15) and subsequently to prove its convergence. Now we give the assumptions on the underlying spaces, involved operators and additional parameters, throughout.

I. Conditions on the underlying spaces:

(UC1): $\mathcal{H}_{1}$ and $\mathcal{H}_{2}$ are two real Hilbert spaces,

(UC2): $\mathcal{C} \subset \mathcal{H}_{1}$ and $\mathcal{Q} \subset \mathcal{H}_{2}$ are two nonempty closed convex sets.

II. Conditions on the involved operators:

(IO1): $\mathcal{A}: \mathcal{H}_{1} \rightarrow \mathcal{H}_{2}$ is a bounded linear operator with its adjoint $\mathcal{A}^{*}$,

(IO2): $\mathcal{B}$ is a strongly positive bounded linear operator on $\mathcal{H}_{1}$ with coefficient

$$
\sigma(>0)
$$

(IO3): $f: \mathcal{C} \rightarrow \mathcal{H}_{1}$ is a $\rho$-contraction,

(IO4): $\mathcal{S}: \mathcal{Q} \rightarrow \mathcal{Q}$ is an $\mathcal{L}_{1}$-Lipschitzian quasi-pseudo-contractive operator with

$\mathcal{L}_{1}(>1)$ and $\mathcal{T}: \mathcal{C} \rightarrow \mathcal{C}$ is an $\mathcal{L}_{2}$-Lipschitzian quasi-pseudo-contractive operator with $\mathcal{L}_{2}(>1)$.

III. Conditions on the parameters:

(AP1): $\delta$ and $\gamma$ are two positive constants,

(AP2): $\left\{\alpha_{n}\right\}_{n \in \mathbb{N}},\left\{\beta_{n}\right\}_{n \in \mathbb{N}},\left\{\gamma_{n}\right\}_{n \in \mathbb{N}},\left\{\zeta_{n}\right\}_{n \in \mathbb{N}}$ and $\left\{\eta_{n}\right\}_{n \in \mathbb{N}}$ are real number sequences in ] $0,1[$.

We use $\Gamma$ to denote the set of solutions of problem (1.15), that is,

$$
\Gamma=\left\{z^{\dagger} \mid z^{\dagger} \in \mathcal{C} \cap \operatorname{Fix}(\mathcal{T}), \mathcal{A} z^{\dagger} \in \mathcal{Q} \cap \operatorname{Fix}(\mathcal{S})\right\}
$$

In the sequel, we assume $\Gamma \neq \emptyset$.

Next, we construct the following iterative algorithm to solve problem (1.15).

Algorithm 3.1 For given $x_{0} \in \mathcal{H}_{1}$ arbitrarily, define a sequence $\left\{x_{n}\right\}$ iteratively by

$$
\left\{\begin{array}{l}
z_{n}=\operatorname{proj}_{\mathcal{Q}} \mathcal{A} x_{n} \\
v_{n}=\left(1-\zeta_{n}\right) z_{n}+\zeta_{n} \mathcal{S}\left(\left(1-\eta_{n}\right) z_{n}+\eta_{n} \mathcal{S} z_{n}\right), \\
y_{n}=\alpha_{n} \gamma f\left(x_{n}\right)+\left(\mathcal{I}-\alpha_{n} \mathcal{B}\right)\left(x_{n}-\delta \mathcal{A}^{*}\left(\mathcal{A} x_{n}-v_{n}\right)\right), \\
u_{n}=\operatorname{proj}_{\mathcal{C}} y_{n} \\
x_{n+1}=\left(1-\beta_{n}\right) u_{n}+\beta_{n} \mathcal{T}\left(\left(1-\gamma_{n}\right) u_{n}+\gamma_{n} \mathcal{T} u_{n}\right)
\end{array}\right.
$$

for all $n \in \mathbb{N}$.

Theorem 3.2 Suppose that $\mathcal{T}-\mathcal{I}$ and $\mathcal{S}-\mathcal{I}$ are demiclosed at 0 . Assume that the following conditions are satisfied:

(C1): $\lim _{n \rightarrow \infty} \alpha_{n}=0$ and $\sum_{n=1}^{\infty} \alpha_{n}=\infty$,

(C2): $0<a_{1}<\zeta_{n}<b_{1}<\eta_{n}<c_{1}<\frac{1}{\sqrt{1+\mathcal{L}_{1}^{2}+1}}$,

(C3): $0<a_{2}<\beta_{n}<b_{2}<\gamma_{n}<c_{2}<\frac{1}{\sqrt{1+\mathcal{L}_{2}^{2}}+1}$,

(C4): $0<\delta<\frac{1}{\|\mathcal{A}\|^{2}}$ and $\sigma>\gamma \rho$. 
Then the sequence $\left\{x_{n}\right\}$ generated by algorithm (3.1) converges strongly to the unique fixed point of the contractive mapping $\operatorname{proj}_{\Gamma}(\gamma f+\mathcal{I}-\mathcal{B})$.

Remark 3.3 In the sequel, we denote the unique fixed point of the mapping $\operatorname{proj}_{\Gamma}(\gamma f+$ $\mathcal{I}-\mathcal{B})$ by $z^{\dagger}$, i.e., $z^{\dagger}=\operatorname{proj}_{\Gamma}(\gamma f+\mathcal{I}-\mathcal{B}) z^{\dagger}$. It is clear that $z^{\dagger}$ solves the variational inequality $\left\langle(\gamma f-\mathcal{B}) z^{\dagger}, z-z^{\dagger}\right\rangle \leq 0, \forall z \in \Gamma$.

In order to prove Theorem 3.2, we need several helpful propositions.

Proposition 3.4 ([19]) Let $\mathcal{H}$ be a real Hilbert space. Let $\mathcal{U}: \mathcal{H} \rightarrow \mathcal{H}$ be an $\mathcal{L}$-Lipschitzian operator with $\mathcal{L}>1$. Then

$$
\operatorname{Fix}(((1-\zeta) \mathcal{I}+\zeta \mathcal{U}) \mathcal{U})=\operatorname{Fix}(\mathcal{U}((1-\zeta) \mathcal{I}+\zeta \mathcal{U}))=\operatorname{Fix}(\mathcal{U})
$$

for all $\zeta \in\left(0, \frac{1}{\mathcal{L}}\right)$.

Proposition 3.5 ([19]) Let $\mathcal{H}$ be a real Hilbert space. Let $\mathcal{U}: \mathcal{H} \rightarrow \mathcal{H}$ be an $\mathcal{L}$-Lipschitzian quasi-pseudo-contractive operator. Then we have

$$
\left\|\mathcal{U}((1-\eta) x+\eta \mathcal{U} x)-u^{\dagger}\right\|^{2} \leq\left\|x-\mathcal{u}^{\dagger}\right\|^{2}+(1-\eta)\|x-\mathcal{U}((1-\eta) x+\eta \mathcal{U} x)\|^{2},
$$

and the operator $(1-\xi) \mathcal{I}+\xi \mathcal{U}((1-\eta) \mathcal{I}+\eta \mathcal{U})$ is quasi-nonexpansive when $0<\xi<\eta<$ $\frac{1}{\sqrt{1+\mathcal{L}^{2}}+1}$, that is,

$$
\left\|(1-\xi) x+\xi \mathcal{U}((1-\eta) x+\eta \mathcal{U} x)-u^{\dagger}\right\| \leq\left\|x-u^{\dagger}\right\|
$$

for all $x \in \mathcal{H}$ and $u^{\dagger} \in \operatorname{Fix}(\mathcal{U})$.

Proposition 3.6 In any real Hilbert space $\mathcal{H}$, the following two equalities hold:

$$
\left\|\zeta u+(1-\zeta) u^{\dagger}\right\|^{2}=\zeta\|u\|^{2}+(1-\zeta)\left\|u^{\dagger}\right\|^{2}-\zeta(1-\zeta)\left\|u-u^{\dagger}\right\|^{2}, \quad \zeta \in[0,1]
$$

and

$$
\left\|u+u^{\dagger}\right\|^{2}=\|u\|^{2}+2\left\langle u, u^{\dagger}\right\rangle+\left\|u^{\dagger}\right\|^{2}
$$

for all $u, u^{\dagger} \in \mathcal{H}$.

Proposition 3.7 ([19]) Let $\mathcal{H}$ be a real Hilbert space. Let $\mathcal{U}: \mathcal{H} \rightarrow \mathcal{H}$ be an $\mathcal{L}$-Lipschitzian operator with $\mathcal{L}>1$. If $\mathcal{I}-\mathcal{U}$ is demiclosed at 0 , then $\mathcal{I}-\mathcal{U}(1-\zeta) \mathcal{I}+\zeta \mathcal{U})$ is also demiclosed at 0 when $\zeta \in\left(0, \frac{1}{\mathcal{L}}\right)$.

Next, we prove Theorem 3.2.

Proof Let $z^{\dagger}=\operatorname{proj}_{\Gamma}(\gamma f+\mathcal{I}-\mathcal{B}) z^{\dagger}$. Subsequently, we obtain $z^{\dagger} \in \mathcal{C} \cap \operatorname{Fix}(\mathcal{T})$ and $\mathcal{A} z^{\dagger} \in$ $\mathcal{Q} \cap \operatorname{Fix}(\mathcal{S})$. Note that $\operatorname{proj}_{\mathcal{Q}}$ is firmly nonexpansive. From (2.1), we deduce

$$
\left\|z_{n}-\mathcal{A} z^{\dagger}\right\|^{2}=\left\|\operatorname{proj}_{\mathcal{Q}} \mathcal{A} x_{n}-\operatorname{proj}_{\mathcal{Q}} \mathcal{A} z^{\dagger}\right\|^{2} \leq\left\|\mathcal{A} x_{n}-\mathcal{A} z^{\dagger}\right\|^{2}-\left\|\mathcal{A} x_{n}-z_{n}\right\|^{2} .
$$


Applying Proposition 3.4 and noting conditions (C2) and (C3), we have

$$
\operatorname{Fix}\left(\mathcal{S}\left(\left(1-\eta_{n}\right) \mathcal{I}+\eta_{n} \mathcal{S}\right)\right)=\operatorname{Fix}(\mathcal{S})
$$

and

$$
\operatorname{Fix}\left(\mathcal{T}\left(\left(1-\gamma_{n}\right) \mathcal{I}+\gamma_{n} \mathcal{T}\right)\right)=\operatorname{Fix}(\mathcal{T})
$$

for all $n \in \mathbb{N}$.

By condition (C2) and Proposition 3.5, we derive

$$
\begin{aligned}
\left\|v_{n}-\mathcal{A} z^{\dagger}\right\|= & \left\|\left[\left(1-\zeta_{n}\right) \mathcal{I}+\zeta_{n} \mathcal{S}\left(\left(1-\eta_{n}\right) \mathcal{I}+\eta_{n} \mathcal{S}\right)\right] z_{n}-\mathcal{A} z^{\dagger}\right\| \\
= & \|\left[\left(1-\zeta_{n}\right) \mathcal{I}+\zeta_{n} \mathcal{S}\left(\left(1-\eta_{n}\right) \mathcal{I}+\eta_{n} \mathcal{S}\right)\right] z_{n} \\
& -\left[\left(1-\zeta_{n}\right) \mathcal{I}+\zeta_{n} \mathcal{S}\left(\left(1-\eta_{n}\right) \mathcal{I}+\eta_{n} \mathcal{S}\right)\right] \mathcal{A} z^{\dagger} \| \\
\leq & \left\|z_{n}-\mathcal{A} z^{\dagger}\right\| .
\end{aligned}
$$

This together with (3.4) implies that

$$
\left\|v_{n}-\mathcal{A} z^{\dagger}\right\|^{2} \leq\left\|\mathcal{A} x_{n}-\mathcal{A} z^{\dagger}\right\|^{2}-\left\|\mathcal{A} x_{n}-z_{n}\right\|^{2} .
$$

By condition (C3) and Proposition 3.5, we derive

$$
\begin{aligned}
\left\|x_{n+1}-z^{\dagger}\right\|= & \left\|\left[\left(1-\beta_{n}\right) \mathcal{I}+\beta_{n} \mathcal{T}\left(\left(1-\gamma_{n}\right) \mathcal{I}+\gamma_{n} \mathcal{T}\right)\right] u_{n}-z^{\dagger}\right\| \\
= & \|\left[\left(1-\beta_{n}\right) \mathcal{I}+\beta_{n} \mathcal{T}\left(\left(1-\gamma_{n}\right) \mathcal{I}+\gamma_{n} \mathcal{T}\right)\right] u_{n} \\
& -\left[\left(1-\beta_{n}\right) \mathcal{I}+\beta_{n} \mathcal{T}\left(\left(1-\gamma_{n}\right) \mathcal{I}+\gamma_{n} \mathcal{T}\right)\right] z^{\dagger} \| \\
\leq & \left\|u_{n}-z^{\dagger}\right\| .
\end{aligned}
$$

Noting that $\operatorname{proj}_{\mathcal{C}}$ is nonexpansive, we obtain

$$
\left\|u_{n}-z^{\dagger}\right\|=\left\|\operatorname{proj}_{\mathcal{C}} y_{n}-\operatorname{proj}_{\mathcal{C}} z^{\dagger}\right\| \leq\left\|y_{n}-z^{\dagger}\right\| .
$$

From (3.1), we get

$$
\begin{aligned}
\left\|y_{n}-z^{\dagger}\right\|= & \left\|\alpha_{n} \gamma f\left(x_{n}\right)+\left(\mathcal{I}-\alpha_{n} \mathcal{B}\right)\left(x_{n}-\delta \mathcal{A}^{*}\left(\mathcal{A} x_{n}-v_{n}\right)\right)-z^{\dagger}\right\| \\
= & \| \alpha_{n} \gamma\left(f\left(x_{n}\right)-f\left(z^{\dagger}\right)\right)+\alpha_{n}\left(\gamma f\left(z^{\dagger}\right)-\mathcal{B} z^{\dagger}\right) \\
& +\left(\mathcal{I}-\alpha_{n} \mathcal{B}\right)\left(x_{n}-z^{\dagger}-\delta \mathcal{A}^{*}\left(\mathcal{A} x_{n}-v_{n}\right)\right) \| \\
\leq & \alpha_{n} \gamma\left\|f\left(x_{n}\right)-f\left(z^{\dagger}\right)\right\|+\alpha_{n}\left\|\gamma f\left(z^{\dagger}\right)-\mathcal{B} z^{\dagger}\right\| \\
& +\left\|\mathcal{I}-\alpha_{n} \mathcal{B}\right\|\left\|x_{n}-z^{\dagger}-\delta \mathcal{A}^{*}\left(\mathcal{A} x_{n}-v_{n}\right)\right\| \\
\leq & \alpha_{n} \gamma \rho\left\|x_{n}-z^{\dagger}\right\|+\alpha_{n}\left\|\gamma f\left(z^{\dagger}\right)-\mathcal{B} z^{\dagger}\right\| \\
& +\left(1-\alpha_{n} \sigma\right)\left\|x_{n}-z^{\dagger}+\delta \mathcal{A}^{*}\left(v_{n}-\mathcal{A} x_{n}\right)\right\| .
\end{aligned}
$$


Observe that

$$
\begin{aligned}
\left\langle x_{n}-z^{\dagger}, \mathcal{A}^{*}\left(v_{n}-\mathcal{A} x_{n}\right)\right\rangle & =\left\langle\mathcal{A}\left(x_{n}-z^{\dagger}\right), v_{n}-\mathcal{A} x_{n}\right\rangle \\
& =\left\langle\mathcal{A} x_{n}-\mathcal{A} z^{\dagger}+v_{n}-\mathcal{A} x_{n}-\left(v_{n}-\mathcal{A} x_{n}\right), v_{n}-\mathcal{A} x_{n}\right\rangle \\
& =\left\langle v_{n}-\mathcal{A} z^{\dagger}, v_{n}-\mathcal{A} x_{n}\right\rangle-\left\|v_{n}-\mathcal{A} x_{n}\right\|^{2} .
\end{aligned}
$$

Using (3.3), we obtain

$$
\left\langle v_{n}-\mathcal{A} z^{\dagger}, v_{n}-\mathcal{A} x_{n}\right\rangle=\frac{1}{2}\left(\left\|v_{n}-\mathcal{A} z^{\dagger}\right\|^{2}+\left\|v_{n}-\mathcal{A} x_{n}\right\|^{2}-\left\|\mathcal{A} x_{n}-\mathcal{A} z^{\dagger}\right\|^{2}\right) .
$$

From (3.5), (3.9) and (3.10), we get

$$
\begin{aligned}
\left\langle x_{n}-z^{\dagger}, \mathcal{A}^{*}\left(v_{n}-\mathcal{A} x_{n}\right)\right\rangle= & \frac{1}{2}\left(\left\|v_{n}-\mathcal{A} z^{\dagger}\right\|^{2}+\left\|v_{n}-\mathcal{A} x_{n}\right\|^{2}-\left\|\mathcal{A} x_{n}-\mathcal{A} z^{\dagger}\right\|^{2}\right) \\
& -\left\|v_{n}-\mathcal{A} x_{n}\right\|^{2} \\
\leq & \frac{1}{2}\left(\left\|\mathcal{A} x_{n}-\mathcal{A} z^{\dagger}\right\|^{2}-\left\|z_{n}-\mathcal{A} x_{n}\right\|^{2}+\left\|v_{n}-\mathcal{A} x_{n}\right\|^{2}\right. \\
& \left.-\left\|\mathcal{A} x_{n}-\mathcal{A} z^{\dagger}\right\|^{2}\right)-\left\|v_{n}-\mathcal{A} x_{n}\right\|^{2} \\
= & -\frac{1}{2}\left\|z_{n}-\mathcal{A} x_{n}\right\|^{2}-\frac{1}{2}\left\|v_{n}-\mathcal{A} x_{n}\right\|^{2} .
\end{aligned}
$$

According to equality (3.3), we get

$$
\begin{aligned}
\left\|x_{n}-z^{\dagger}+\delta \mathcal{A}^{*}\left(v_{n}-\mathcal{A} x_{n}\right)\right\|^{2}= & \left\|x_{n}-z^{\dagger}\right\|^{2}+\delta^{2}\left\|\mathcal{A}^{*}\left(v_{n}-\mathcal{A} x_{n}\right)\right\|^{2} \\
& +2 \delta\left(x_{n}-z^{\dagger}, \mathcal{A}^{*}\left(v_{n}-\mathcal{A} x_{n}\right)\right) .
\end{aligned}
$$

Combining the above equality and (3.11), we deduce

$$
\begin{aligned}
\left\|x_{n}-z^{\dagger}+\delta \mathcal{A}^{*}\left(v_{n}-\mathcal{A} x_{n}\right)\right\|^{2} \leq & \left\|x_{n}-z^{\dagger}\right\|^{2}+\delta^{2}\|\mathcal{A}\|^{2}\left\|v_{n}-\mathcal{A} x_{n}\right\|^{2} \\
& -\delta\left\|z_{n}-\mathcal{A} x_{n}\right\|^{2}-\delta\left\|v_{n}-\mathcal{A} x_{n}\right\|^{2} \\
= & \left\|x_{n}-z^{\dagger}\right\|^{2}+\left(\delta^{2}\|\mathcal{A}\|^{2}-\delta\right)\left\|v_{n}-\mathcal{A} x_{n}\right\|^{2} \\
& -\delta\left\|z_{n}-\mathcal{A} x_{n}\right\|^{2} .
\end{aligned}
$$

In view of condition (C4), we know that $\delta^{2}\|\mathcal{A}\|^{2}-\delta<0$. From (3.12), we have

$$
\left\|x_{n}-z^{\dagger}+\delta \mathcal{A}^{*}\left(v_{n}-\mathcal{A} x_{n}\right)\right\|^{2} \leq\left\|x_{n}-z^{\dagger}\right\|^{2}
$$

Therefore,

$$
\left\|x_{n}-z^{\dagger}+\delta \mathcal{A}^{*}\left(v_{n}-\mathcal{A} x_{n}\right)\right\| \leq\left\|x_{n}-z^{\dagger}\right\|
$$

Substituting (3.13) into (3.8) we deduce

$$
\begin{aligned}
\left\|y_{n}-z^{\dagger}\right\| & \leq \alpha_{n} \gamma \rho\left\|x_{n}-z^{\dagger}\right\|+\alpha_{n}\left\|\gamma f\left(z^{\dagger}\right)-\mathcal{B} z^{\dagger}\right\|+\left(1-\alpha_{n} \sigma\right)\left\|x_{n}-z^{\dagger}\right\| \\
& =\left[1-(\sigma-\gamma \rho) \alpha_{n}\right]\left\|x_{n}-z^{\dagger}\right\|+\alpha_{n}\left\|\gamma f\left(z^{\dagger}\right)-\mathcal{B} z^{\dagger}\right\| .
\end{aligned}
$$


From (3.6), (3.7) and (3.14), we get

$$
\begin{aligned}
\left\|x_{n+1}-z^{\dagger}\right\| & \leq\left[1-(\sigma-\gamma \rho) \alpha_{n}\right]\left\|x_{n}-z^{\dagger}\right\|+\alpha_{n}\left\|\gamma f\left(z^{\dagger}\right)-\mathcal{B} z^{\dagger}\right\| \\
& =\left[1-(\sigma-\gamma \rho) \alpha_{n}\right]\left\|x_{n}-z^{\dagger}\right\|+(\sigma-\gamma \rho) \alpha_{n} \frac{\left\|\gamma f\left(z^{\dagger}\right)-\mathcal{B} z^{\dagger}\right\|}{\sigma-\gamma \rho} .
\end{aligned}
$$

By induction, we get

$$
\left\|x_{n}-z^{\dagger}\right\| \leq \max \left\{\left\|x_{0}-z^{\dagger}\right\|, \frac{\left\|\gamma f\left(z^{\dagger}\right)-\mathcal{B} z^{\dagger}\right\|}{\sigma-\gamma \rho}\right\} .
$$

Hence, the sequence $\left\{x_{n}\right\}$ is bounded.

Using the firm nonexpansiveness of $\operatorname{proj}_{\mathcal{C}}$, we have

$$
\begin{aligned}
\left\|u_{n}-z^{\dagger}\right\|^{2} & =\left\|\operatorname{proj}_{\mathcal{C}} y_{n}-z^{\dagger}\right\|^{2} \\
& \leq\left\|y_{n}-z^{\dagger}\right\|^{2}-\left\|\operatorname{proj}_{\mathcal{C}} y_{n}-y_{n}\right\|^{2} \\
& =\left\|y_{n}-z^{\dagger}\right\|^{2}-\left\|u_{n}-y_{n}\right\|^{2}
\end{aligned}
$$

From (3.6), (3.14) and (3.15), we deduce

$$
\begin{aligned}
\left\|x_{n+1}-z^{\dagger}\right\|^{2} & \leq\left\|u_{n}-z^{\dagger}\right\|^{2} \\
& \leq\left\|y_{n}-z^{\dagger}\right\|^{2}-\left\|u_{n}-y_{n}\right\|^{2} \\
& \leq\left[1-(\sigma-\gamma \rho) \alpha_{n}\right]\left\|x_{n}-z^{\dagger}\right\|^{2}+\frac{\alpha_{n}}{\sigma-\gamma \rho}\left\|\gamma f\left(z^{\dagger}\right)-\mathcal{B} z^{\dagger}\right\|^{2}-\left\|u_{n}-y_{n}\right\|^{2} .
\end{aligned}
$$

It follows that

$$
\left\|u_{n}-y_{n}\right\|^{2} \leq\left\|x_{n}-z^{\dagger}\right\|^{2}-\left\|x_{n+1}-z^{\dagger}\right\|^{2}+\frac{\alpha_{n}}{\sigma-\gamma \rho}\left\|\gamma f\left(z^{\dagger}\right)-\mathcal{B} z^{\dagger}\right\|^{2}
$$

Next, we consider two possible cases: the sequence $\left\{\left\|x_{n}-z^{\dagger}\right\|\right\}$ is either monotone decreasing at infinity (Case 1 ) or not (Case 2).

Case 1. There exists $n_{0}$ such that the sequence $\left\{\left\|x_{n}-z^{\dagger}\right\|\right\}_{n \geq n_{0}}$ is decreasing.

Case 2. For any $n_{0}$, there exists an integer $m \geq n_{0}$ such that $\left\|x_{m}-z^{\dagger}\right\| \leq\left\|x_{m+1}-z^{\dagger}\right\|$.

In Case 1 , we assume that there exists some integer $m>0$ such that $\left\{\left\|x_{n}-z^{\dagger}\right\|\right\}$ is decreasing for all $n \geq m$. Then $\lim _{n \rightarrow \infty}\left\|x_{n}-z^{\dagger}\right\|$ exists. From (3.16), we deduce

$$
\lim _{n \rightarrow \infty}\left\|u_{n}-y_{n}\right\|=0 .
$$

From (3.8), we have

$$
\begin{aligned}
\left\|y_{n}-z^{\dagger}\right\| \leq & \alpha_{n} \gamma \rho\left\|x_{n}-z^{\dagger}\right\|+\alpha_{n}\left\|\gamma f\left(z^{\dagger}\right)-\mathcal{B} z^{\dagger}\right\| \\
& +\left(1-\alpha_{n} \sigma\right)\left\|x_{n}-z^{\dagger}+\delta \mathcal{A}^{*}\left(v_{n}-\mathcal{A} x_{n}\right)\right\| \\
= & \alpha_{n} \sigma \frac{\gamma \rho\left\|x_{n}-z^{\dagger}\right\|+\left\|\gamma f\left(z^{\dagger}\right)-\mathcal{B} z^{\dagger}\right\|}{\sigma} \\
& +\left(1-\alpha_{n} \sigma\right)\left\|x_{n}-z^{\dagger}+\delta \mathcal{A}^{*}\left(v_{n}-\mathcal{A} x_{n}\right)\right\| .
\end{aligned}
$$


Since $\left\{x_{n}\right\}$ is bounded, there exists a constant $M>$ such that

$$
\sup _{n}\left\{\frac{\gamma \rho\left\|x_{n}-z^{\dagger}\right\|+\left\|\gamma f\left(z^{\dagger}\right)-\mathcal{B} z^{\dagger}\right\|}{\sigma}\right\}<M .
$$

By (3.18), we deduce

$$
\left\|y_{n}-z^{\dagger}\right\|^{2} \leq \alpha_{n} \sigma M^{2}+\left(1-\alpha_{n} \sigma\right)\left\|x_{n}-z^{\dagger}+\delta \mathcal{A}^{*}\left(v_{n}-\mathcal{A} x_{n}\right)\right\|^{2} .
$$

Combining (3.12) and (3.19), we obtain

$$
\begin{aligned}
\left\|x_{n+1}-z^{\dagger}\right\|^{2} \leq & \left\|y_{n}-z^{\dagger}\right\|^{2} \\
\leq & \left(1-\sigma \alpha_{n}\right)\left\|x_{n}-z^{\dagger}\right\|^{2}+\left(1-\sigma \alpha_{n}\right)\left(\delta^{2}\|\mathcal{A}\|^{2}-\delta\right)\left\|v_{n}-\mathcal{A} x_{n}\right\|^{2} \\
& -\left(1-\sigma \alpha_{n}\right) \delta\left\|z_{n}-\mathcal{A} x_{n}\right\|^{2}+\alpha_{n} \sigma M^{2} .
\end{aligned}
$$

Hence,

$$
\begin{aligned}
0 & \leq\left(1-\sigma \alpha_{n}\right)\left(\delta-\delta^{2}\|\mathcal{A}\|^{2}\right)\left\|v_{n}-\mathcal{A} x_{n}\right\|^{2}+\left(1-\sigma \alpha_{n}\right) \delta\left\|z_{n}-\mathcal{A} x_{n}\right\|^{2} \\
& \leq\left(1-\sigma \alpha_{n}\right)\left\|x_{n}-z^{\dagger}\right\|^{2}-\left\|x_{n+1}-z^{\dagger}\right\|^{2}+\alpha_{n} \sigma M^{2}
\end{aligned}
$$

which implies that

$$
\lim _{n \rightarrow \infty}\left\|v_{n}-\mathcal{A} x_{n}\right\|=\lim _{n \rightarrow \infty}\left\|z_{n}-\mathcal{A} x_{n}\right\|=0 .
$$

Therefore,

$$
\lim _{n \rightarrow \infty}\left\|v_{n}-z_{n}\right\|=0
$$

Note that $v_{n}-z_{n}=\zeta_{n}\left[\mathcal{S}\left(\left(1-\eta_{n}\right) \mathcal{I}+\eta_{n} \mathcal{S}\right) z_{n}-z_{n}\right]$. Thus,

$$
\lim _{n \rightarrow \infty}\left\|z_{n}-\mathcal{S}\left(\left(1-\eta_{n}\right) \mathcal{I}+\eta_{n} \mathcal{S}\right) z_{n}\right\|=\lim _{n \rightarrow \infty}\left\|\mathcal{A} x_{n}-\mathcal{S}\left(\left(1-\eta_{n}\right) \mathcal{I}+\eta_{n} \mathcal{S}\right) \mathcal{A} x_{n}\right\|=0
$$

Since

$$
\begin{aligned}
\left\|\mathcal{A} x_{n}-\mathcal{S} \mathcal{A} x_{n}\right\| \leq & \left\|\mathcal{A} x_{n}-S\left(\left(1-\eta_{n}\right) \mathcal{I}+\eta_{n} \mathcal{S}\right) \mathcal{A} x_{n}\right\| \\
& +\left\|\mathcal{S}\left(\left(1-\eta_{n}\right) \mathcal{I}+\eta_{n} \mathcal{S}\right) \mathcal{A} x_{n}-\mathcal{S} \mathcal{A} x_{n}\right\| \\
\leq & \left\|\mathcal{A} x_{n}-\mathcal{S}\left(\left(1-\eta_{n}\right) \mathcal{I}+\eta_{n} \mathcal{S}\right) \mathcal{A} x_{n}\right\|+\mathcal{L}_{1} \eta_{n}\left\|\mathcal{A} x_{n}-\mathcal{S} \mathcal{A} x_{n}\right\|,
\end{aligned}
$$

it follows that

$$
\left\|\mathcal{A} x_{n}-\mathcal{S} \mathcal{A} x_{n}\right\| \leq \frac{1}{1-\mathcal{L}_{1} \eta_{n}}\left\|\mathcal{A} x_{n}-\mathcal{S}\left(\left(1-\eta_{n}\right) \mathcal{I}+\eta_{n} \mathcal{S}\right) \mathcal{A} x_{n}\right\|
$$

This together with (3.22) implies that

$$
\lim _{n \rightarrow \infty}\left\|\mathcal{A} x_{n}-\mathcal{S} \mathcal{A} x_{n}\right\|=0 .
$$


According to (3.1), we have

$$
\begin{aligned}
\left\|y_{n}-x_{n}\right\| & =\left\|\alpha_{n} \gamma f\left(x_{n}\right)-\delta \mathcal{A}^{*}\left(\mathcal{A} x_{n}-v_{n}\right)-\alpha_{n} \mathcal{B}\left(x_{n}-\delta \mathcal{A}^{*}\left(\mathcal{A} x_{n}-v_{n}\right)\right)\right\| \\
& \leq \delta\|\mathcal{A}\|\left\|v_{n}-\mathcal{A} x_{n}\right\|+\alpha_{n}\left\|\gamma f\left(x_{n}\right)-\mathcal{B}\left(x_{n}-\delta \mathcal{A}^{*}\left(\mathcal{A} x_{n}-v_{n}\right)\right)\right\|
\end{aligned}
$$

It follows from (3.20) and (C1) that

$$
\lim _{n \rightarrow \infty}\left\|x_{n}-y_{n}\right\|=0
$$

From (3.1) and (3.2), we have

$$
\begin{aligned}
\left\|x_{n+1}-z^{\dagger}\right\|^{2}= & \left\|\left(1-\beta_{n}\right)\left(u_{n}-z^{\dagger}\right)+\beta_{n}\left[\mathcal{T}\left(\left(1-\gamma_{n}\right) u_{n}+\gamma_{n} \mathcal{T} u_{n}\right)-z^{\dagger}\right]\right\|^{2} \\
= & \left(1-\beta_{n}\right)\left\|u_{n}-z^{\dagger}\right\|^{2}+\beta_{n}\left\|\mathcal{T}\left(\left(1-\gamma_{n}\right) u_{n}+\gamma_{n} \mathcal{T} u_{n}\right)-z^{\dagger}\right\|^{2} \\
& -\beta_{n}\left(1-\beta_{n}\right)\left\|\mathcal{T}\left(\left(1-\gamma_{n}\right) u_{n}+\gamma_{n} \mathcal{T} u_{n}\right)-u_{n}\right\|^{2} .
\end{aligned}
$$

Applying Proposition 3.5, we get

$$
\begin{aligned}
& \left\|\mathcal{T}\left(\left(1-\gamma_{n}\right) u_{n}+\gamma_{n} \mathcal{T} u_{n}\right)-z^{\dagger}\right\|^{2} \\
& \quad \leq\left\|u_{n}-z^{\dagger}\right\|^{2}+\left(1-\gamma_{n}\right)\left\|u_{n}-\mathcal{T}\left(\left(1-\gamma_{n}\right) u_{n}+\gamma_{n} \mathcal{T} u_{n}\right)\right\|^{2}
\end{aligned}
$$

From (3.19), (3.25) and (3.26), we deduce

$$
\begin{aligned}
\left\|x_{n+1}-z^{\dagger}\right\|^{2} \leq & \left\|u_{n}-z^{\dagger}\right\|^{2}-\beta_{n}\left(\gamma_{n}-\beta_{n}\right)\left\|u_{n}-\mathcal{T}\left(\left(1-\gamma_{n}\right) u_{n}+\gamma_{n} \mathcal{T} u_{n}\right)\right\|^{2} \\
\leq & \alpha_{n} \sigma M^{2}+\left(1-\alpha_{n} \sigma\right)\left\|x_{n}-z^{\dagger}+\delta \mathcal{A}^{*}\left(v_{n}-\mathcal{A} x_{n}\right)\right\|^{2} \\
& \quad-\beta_{n}\left(\gamma_{n}-\beta_{n}\right)\left\|u_{n}-\mathcal{T}\left(\left(1-\gamma_{n}\right) u_{n}+\gamma_{n} \mathcal{T} u_{n}\right)\right\|^{2} \\
\leq & \alpha_{n} \sigma M^{2}+\left\|x_{n}-z^{\dagger}\right\|^{2}-\beta_{n}\left(\gamma_{n}-\beta_{n}\right)\left\|u_{n}-\mathcal{T}\left(\left(1-\gamma_{n}\right) u_{n}+\gamma_{n} \mathcal{T} u_{n}\right)\right\|^{2} .
\end{aligned}
$$

It follows that

$$
\begin{gathered}
\beta_{n}\left(\gamma_{n}-\beta_{n}\right)\left\|u_{n}-\mathcal{T}\left(\left(1-\gamma_{n}\right) u_{n}+\gamma_{n} \mathcal{T} u_{n}\right)\right\|^{2} \\
\leq\left\|x_{n}-z^{\dagger}\right\|^{2}-\left\|x_{n+1}-z^{\dagger}\right\|^{2}+\alpha_{n} \sigma M^{2} .
\end{gathered}
$$

Therefore,

$$
\lim _{n \rightarrow \infty}\left\|u_{n}-\mathcal{T}\left(\left(1-\gamma_{n}\right) u_{n}+\gamma_{n} \mathcal{T} u_{n}\right)\right\|=0
$$

Observe that

$$
\begin{aligned}
\left\|u_{n}-\mathcal{T} u_{n}\right\| & \leq\left\|u_{n}-\mathcal{T}\left(\left(1-\gamma_{n}\right) u_{n}+\gamma_{n} \mathcal{T} u_{n}\right)\right\|+\left\|\mathcal{T}\left(\left(1-\gamma_{n}\right) u_{n}+\gamma_{n} \mathcal{T} u_{n}\right)-\mathcal{T} u_{n}\right\| \\
& \leq\left\|u_{n}-\mathcal{T}\left(\left(1-\gamma_{n}\right) u_{n}+\gamma_{n} \mathcal{T} u_{n}\right)\right\|+\mathcal{L}_{2} \gamma_{n}\left\|u_{n}-\mathcal{T} u_{n}\right\| .
\end{aligned}
$$

Thus,

$$
\left\|u_{n}-\mathcal{T} u_{n}\right\| \leq \frac{1}{1-\mathcal{L}_{2} \gamma_{n}}\left\|u_{n}-\mathcal{T}\left(\left(1-\gamma_{n}\right) u_{n}+\gamma_{n} \mathcal{T} u_{n}\right)\right\| .
$$


This together with (3.27) implies that

$$
\lim _{n \rightarrow \infty}\left\|u_{n}-\mathcal{T} u_{n}\right\|=0
$$

Next, we show that

$$
\limsup _{n \rightarrow \infty}\left\langle\gamma f\left(z^{\dagger}\right)-\mathcal{B} z^{\dagger}, y_{n}-z^{\dagger}\right\rangle \leq 0 .
$$

Choose a subsequence $\left\{y_{n_{i}}\right\}$ of $\left\{y_{n}\right\}$ such that

$$
\limsup _{n \rightarrow \infty}\left\langle\gamma f\left(z^{\dagger}\right)-\mathcal{B} z^{\dagger}, y_{n}-z^{\dagger}\right\rangle=\lim _{i \rightarrow \infty}\left\langle\gamma f\left(z^{\dagger}\right)-\mathcal{B} z^{\dagger}, y_{n_{i}}-z^{\dagger}\right\rangle .
$$

Since the sequence $\left\{y_{n_{i}}\right\}$ is bounded, we can choose a subsequence $\left\{y_{n_{i j}}\right\}$ of $\left\{y_{n_{i}}\right\}$ such that $y_{n_{i_{j}}} \rightarrow z$. For the sake of convenience, we assume (without loss of generality) that $y_{n_{i}} \rightarrow z$. Subsequently, we derive from the above conclusions that

$$
\left\{\begin{array}{l}
x_{n_{i}}-z, \\
y_{n_{i}}-z, \\
u_{n_{i}}-z
\end{array}\right.
$$

and

$$
\left\{\begin{array}{l}
\mathcal{A} x_{n_{i}} \rightarrow \mathcal{A} z, \\
\mathcal{A} y_{n_{i}} \rightarrow \mathcal{A} z, \\
\mathcal{A} u_{n_{i}} \rightarrow \mathcal{A} z .
\end{array}\right.
$$

Note that $u_{n_{i}}=\operatorname{proj}_{\mathcal{C}} y_{n_{i}} \in \mathcal{C}$ and $z_{n_{i}}=\operatorname{proj}_{\mathcal{Q}} \mathcal{A} x_{n_{i}} \in \mathcal{Q}$. From (3.30), we deduce $z \in \mathcal{C}$ and $\mathcal{A} z \in \mathcal{Q}$ by (3.31). By the demiclosedness of $\mathcal{T}-\mathcal{I}$ and $\mathcal{S}-\mathcal{I}$, we deduce $z \in \operatorname{Fix}(\mathcal{T})$ (by (3.28)) and $\mathcal{A} z \in \operatorname{Fix}(\mathcal{S})$ (by (3.23)). To this end, we deduce $z \in \mathcal{C} \cap \operatorname{Fix}(\mathcal{T})$ and $\mathcal{A} z \in \mathcal{Q} \cap$ $\operatorname{Fix}(\mathcal{S})$. That is to say, $z \in \Gamma$.

Therefore,

$$
\begin{aligned}
\limsup _{n \rightarrow \infty}\left\langle\gamma f\left(z^{\dagger}\right)-\mathcal{B} z^{\dagger}, y_{n}-z^{\dagger}\right\rangle & =\lim _{i \rightarrow \infty}\left\langle\gamma f\left(z^{\dagger}\right)-\mathcal{B} z^{\dagger}, y_{n_{i}}-z^{\dagger}\right\rangle \\
& =\lim _{i \rightarrow \infty}\left\langle\gamma f\left(z^{\dagger}\right)-\mathcal{B} z^{\dagger}, z-z^{\dagger}\right\rangle \\
& \leq 0 .
\end{aligned}
$$

From (3.1), we have

$$
\begin{aligned}
\left\|y_{n}-z^{\dagger}\right\|^{2}= & \| \alpha_{n} \gamma\left(f\left(x_{n}\right)-f\left(z^{\dagger}\right)\right)+\alpha_{n}\left(\gamma f\left(z^{\dagger}\right)-\mathcal{B} z^{\dagger}\right) \\
& +\left(\mathcal{I}-\alpha_{n} \mathcal{B}\right)\left(x_{n}-z^{\dagger}-\delta \mathcal{A}^{*}\left(\mathcal{A} x_{n}-v_{n}\right)\right) \|^{2} \\
\leq & \left\|\mathcal{I}-\alpha_{n} \mathcal{B}\right\|^{2}\left\|x_{n}-z^{\dagger}-\delta \mathcal{A}^{*}\left(\mathcal{A} x_{n}-v_{n}\right)\right\|^{2} \\
& +2 \alpha_{n} \gamma\left\langle f\left(x_{n}\right)-f\left(z^{\dagger}\right), y_{n}-z^{\dagger}\right\rangle+2 \alpha_{n}\left\langle\gamma f\left(z^{\dagger}\right)-\mathcal{B} z^{\dagger}, y_{n}-z^{\dagger}\right\rangle
\end{aligned}
$$




$$
\begin{aligned}
\leq & \left(1-\alpha_{n} \sigma\right)^{2}\left\|x_{n}-z^{\dagger}\right\|^{2}+2 \alpha_{n} \gamma\left\|f\left(x_{n}\right)-f\left(z^{\dagger}\right)\right\|\left\|y_{n}-z^{\dagger}\right\| \\
& +2 \alpha_{n}\left\langle\gamma f\left(z^{\dagger}\right)-\mathcal{B} z^{\dagger}, y_{n}-z^{\dagger}\right\rangle \\
\leq & \left(1-\alpha_{n} \sigma\right)^{2}\left\|x_{n}-z^{\dagger}\right\|^{2}+\alpha_{n} \gamma \rho\left\|x_{n}-z^{\dagger}\right\|^{2}+\alpha_{n} \gamma \rho\left\|y_{n}-z^{\dagger}\right\|^{2} \\
& +2 \alpha_{n}\left\langle\gamma f\left(z^{\dagger}\right)-\mathcal{B} z^{\dagger}, y_{n}-z^{\dagger}\right\rangle .
\end{aligned}
$$

It follows that

$$
\begin{aligned}
\left\|y_{n}-z^{\dagger}\right\|^{2} \leq & {\left[1-\frac{2(\sigma-\gamma \rho) \alpha_{n}}{1-\gamma \rho \alpha_{n}}\right]\left\|x_{n}-z^{\dagger}\right\|^{2}+\frac{\sigma^{2} \alpha_{n}^{2}}{1-\gamma \rho \alpha_{n}}\left\|x_{n}-z^{\dagger}\right\|^{2} } \\
& +\frac{2 \alpha_{n}}{1-\gamma \rho \alpha_{n}}\left\langle\gamma f\left(z^{\dagger}\right)-\mathcal{B} z^{\dagger}, y_{n}-z^{\dagger}\right\rangle .
\end{aligned}
$$

Therefore,

$$
\begin{aligned}
\left\|x_{n+1}-z^{\dagger}\right\|^{2} \leq & \left\|y_{n}-z^{\dagger}\right\|^{2} \\
\leq & {\left[1-\frac{2(\sigma-\gamma \rho) \alpha_{n}}{1-\gamma \rho \alpha_{n}}\right]\left\|x_{n}-z^{\dagger}\right\|^{2}+\frac{\sigma^{2} \alpha_{n}^{2}}{1-\gamma \rho \alpha_{n}}\left\|x_{n}-z^{\dagger}\right\|^{2} } \\
& +\frac{2 \alpha_{n}}{1-\gamma \rho \alpha_{n}}\left\langle\gamma f\left(z^{\dagger}\right)-\mathcal{B} z^{\dagger}, y_{n}-z^{\dagger}\right\rangle .
\end{aligned}
$$

Applying Lemma 2.6 and (3.32) to (3.33), we deduce $x_{n} \rightarrow z^{\dagger}$.

Case 2. Assume that there exists an integer $n_{0}$ such that

$$
\left\|x_{n_{0}}-z^{\dagger}\right\| \leq\left\|x_{n_{0}+1}-z^{\dagger}\right\|
$$

Set $\omega_{n}=\left\{\left\|x_{n}-z^{\dagger}\right\|\right\}$. Then we have

$$
\omega_{n_{0}} \leq \omega_{n_{0}+1}
$$

Define an integer sequence $\left\{\tau_{n}\right\}$ for all $n \geq n_{0}$ as follows:

$$
\tau(n)=\max \left\{l \in \mathbb{N} \mid n_{0} \leq l \leq n, \omega_{l} \leq \omega_{l+1}\right\}
$$

It is clear that $\tau(n)$ is a nondecreasing sequence satisfying

$$
\lim _{n \rightarrow \infty} \tau(n)=\infty
$$

and

$$
\omega_{\tau(n)} \leq \omega_{\tau(n)+1}
$$

for all $n \geq n_{0}$.

By a similar argument as that of Case 1, we can obtain

$$
\begin{aligned}
& \lim _{n \rightarrow \infty}\left\|u_{\tau(n)}-y_{\tau(n)}\right\|=\lim _{n \rightarrow \infty}\left\|x_{\tau(n)}-y_{\tau(n)}\right\|=0, \\
& \lim _{n \rightarrow \infty}\left\|\mathcal{S} x_{\tau(n)}-\mathcal{A} x_{\tau(n)}\right\|=0
\end{aligned}
$$


and

$$
\lim _{n \rightarrow \infty}\left\|u_{\tau(n)}-\mathcal{T} u_{\tau(n)}\right\|=0 .
$$

This implies that

$$
\omega_{w}\left(y_{\tau(n)}\right) \subset \Gamma .
$$

Thus, we obtain

$$
\limsup _{n \rightarrow \infty}\left\langle\gamma f\left(z^{\dagger}\right)-\mathcal{B} z^{\dagger}, y_{\tau(n)}-z^{\dagger}\right\rangle \leq 0
$$

Since $\omega_{\tau(n)} \leq \omega_{\tau(n)+1}$, we have from (3.33) that

$$
\begin{aligned}
\omega_{\tau(n)}^{2} \leq & \omega_{\tau(n)+1}^{2} \\
\leq & {\left[1-\frac{2(\sigma-\gamma \rho) \alpha_{\tau(n)}}{1-\gamma \rho \alpha_{\tau(n)}}\right] \omega_{\tau(n)}^{2}+\frac{\sigma^{2} \alpha_{\tau(n)}^{2}}{1-\gamma \rho \alpha_{\tau(n)}} \omega_{\tau(n)}^{2} } \\
& +\frac{2 \alpha_{\tau(n)}}{1-\gamma \rho \alpha_{\tau(n)}}\left\langle\gamma f\left(z^{\dagger}\right)-\mathcal{B} z^{\dagger}, y_{\tau(n)}-z^{\dagger}\right\rangle .
\end{aligned}
$$

It follows that

$$
\omega_{\tau(n)}^{2} \leq \frac{2}{2(\sigma-\gamma \rho)-\sigma^{2} \alpha_{\tau(n)}}\left\langle\gamma f\left(z^{\dagger}\right)-\mathcal{B} z^{\dagger}, y_{\tau(n)}-z^{\dagger}\right\rangle .
$$

Combining (3.34) and (3.36), we have

$$
\limsup _{n \rightarrow \infty} \omega_{\tau(n)} \leq 0
$$

and hence

$$
\lim _{n \rightarrow \infty} \omega_{\tau(n)}=0 .
$$

By (3.35), we obtain

$$
\limsup _{n \rightarrow \infty} \omega_{\tau(n)+1}^{2} \leq \limsup _{n \rightarrow \infty} \omega_{\tau(n)}^{2}
$$

This together with (3.37) implies that

$$
\lim _{n \rightarrow \infty} \omega_{\tau(n)+1}=0
$$

Applying Lemma 2.7 we get

$$
0 \leq \omega_{n} \leq \max \left\{\omega_{\tau(n)}, \omega_{\tau(n)+1}\right\}
$$

Therefore, $\omega_{n} \rightarrow 0$. That is, $x_{n} \rightarrow z^{\dagger}$. This completes the proof. 


\section{Applications}

The following results can be deduced directly from Algorithm 3.1 and Theorem 3.2.

Algorithm 4.1 For given $x_{0} \in \mathcal{H}_{1}$ arbitrarily, define a sequence $\left\{x_{n}\right\}$ iteratively by

$$
\left\{\begin{array}{l}
z_{n}=\operatorname{proj}_{\mathcal{Q}} \mathcal{A} x_{n}, \\
v_{n}=\left(1-\zeta_{n}\right) z_{n}+\zeta_{n} \mathcal{S}\left(\left(1-\eta_{n}\right) z_{n}+\eta_{n} \mathcal{S} z_{n}\right), \\
y_{n}=\left(1-\alpha_{n}\right)\left(x_{n}-\delta \mathcal{A}^{*}\left(\mathcal{A} x_{n}-v_{n}\right)\right) \\
u_{n}=\operatorname{proj}_{\mathcal{C}} y_{n}, \\
x_{n+1}=\left(1-\beta_{n}\right) u_{n}+\beta_{n} \mathcal{T}\left(\left(1-\gamma_{n}\right) u_{n}+\gamma_{n} \mathcal{T} u_{n}\right)
\end{array}\right.
$$

for all $n \in \mathbb{N}$.

Corollary 4.2 Suppose that $\mathcal{T}-\mathcal{I}$ and $\mathcal{S}-\mathcal{I}$ are demiclosed at 0 . Assume that the following conditions are satisfied:

(C1): $\lim _{n \rightarrow \infty} \alpha_{n}=0$ and $\sum_{n=1}^{\infty} \alpha_{n}=\infty$,

(C2): $0<a_{1}<\zeta_{n}<b_{1}<\eta_{n}<c_{1}<\frac{1}{\sqrt{1+\mathcal{L}_{1}^{2}+1}}$,

(C3): $0<a_{2}<\beta_{n}<b_{2}<\gamma_{n}<c_{2}<\frac{1}{\sqrt{1+\mathcal{L}_{2}^{2}+1}}$,

$(\mathrm{C} 4)^{\prime}: 0<\delta<\frac{1}{\|\mathcal{A}\|^{2}}$.

Then the sequence $\left\{x_{n}\right\}$ generated by algorithm (4.1) converges strongly to the minimum norm solution $u^{*} \in \Gamma$.

Algorithm 4.3 For given $x_{0} \in \mathcal{H}_{1}$ arbitrarily, define a sequence $\left\{x_{n}\right\}$ iteratively by

$$
x_{n+1}=\operatorname{proj}_{\mathcal{C}}\left[\alpha_{n} \gamma f\left(x_{n}\right)+\left(\mathcal{I}-\alpha_{n} \mathcal{B}\right)\left(x_{n}-\delta \mathcal{A}^{*}\left(\mathcal{A} x_{n}-\operatorname{proj}_{\mathcal{Q}} \mathcal{A} x_{n}\right)\right)\right]
$$

for all $n \in \mathbb{N}$.

Corollary 4.4 Assume that the following conditions are satisfied:

(C1): $\lim _{n \rightarrow \infty} \alpha_{n}=0$ and $\sum_{n=1}^{\infty} \alpha_{n}=\infty$,

(C4): $0<\delta<\frac{1}{\|\mathcal{A}\|^{2}}$ and $\sigma>\gamma \rho$.

Then the sequence $\left\{x_{n}\right\}$ generated by algorithm (4.2) converges strongly to $u \in \Gamma_{1}$ (the set of the solutions of (1.1)) provided $\Gamma_{1} \neq \emptyset$.

Algorithm 4.5 For given $x_{0} \in \mathcal{H}_{1}$ arbitrarily, define a sequence $\left\{x_{n}\right\}$ iteratively by

$$
x_{n+1}=\operatorname{proj}_{\mathcal{C}}\left[\left(1-\alpha_{n}\right)\left(x_{n}-\delta \mathcal{A}^{*}\left(\mathcal{A} x_{n}-\operatorname{proj}_{\mathcal{Q}} \mathcal{A} x_{n}\right)\right)\right]
$$

for all $n \in \mathbb{N}$.

Corollary 4.6 Assume that the following conditions are satisfied:

(C1): $\lim _{n \rightarrow \infty} \alpha_{n}=0$ and $\sum_{n=1}^{\infty} \alpha_{n}=\infty$,

$(\mathrm{C} 4)^{\prime}: 0<\delta<\frac{1}{\|\mathcal{A}\|^{2}}$. 
Then the sequence $\left\{x_{n}\right\}$ generated by algorithm (4.3) converges strongly to the minimum norm solution $u^{\boldsymbol{*}} \in \Gamma_{1}$ provided $\Gamma_{1} \neq \emptyset$.

Algorithm 4.7 For given $x_{0} \in \mathcal{H}_{1}$ arbitrarily, define a sequence $\left\{x_{n}\right\}$ iteratively by

$$
\left\{\begin{array}{l}
v_{n}=\left(1-\zeta_{n}\right) \mathcal{A} x_{n}+\zeta_{n} \mathcal{S}\left(\left(1-\eta_{n}\right) \mathcal{A} x_{n}+\eta_{n} \mathcal{S} \mathcal{A} x_{n}\right), \\
y_{n}=\alpha_{n} \gamma f\left(x_{n}\right)+\left(\mathcal{I}-\alpha_{n} \mathcal{B}\right)\left(x_{n}-\delta \mathcal{A}^{*}\left(\mathcal{A} x_{n}-v_{n}\right)\right), \\
x_{n+1}=\left(1-\beta_{n}\right) y_{n}+\beta_{n} \mathcal{T}\left(\left(1-\gamma_{n}\right) y_{n}+\gamma_{n} \mathcal{T} y_{n}\right)
\end{array}\right.
$$

for all $n \in \mathbb{N}$.

Corollary 4.8 Suppose that $\mathcal{T}-\mathcal{I}$ and $\mathcal{S}-\mathcal{I}$ are demiclosed at 0 . Assume that the following conditions are satisfied:

(C1): $\lim _{n \rightarrow \infty} \alpha_{n}=0$ and $\sum_{n=1}^{\infty} \alpha_{n}=\infty$,

(C2): $0<a_{1}<\zeta_{n}<b_{1}<\eta_{n}<c_{1}<\frac{1}{\sqrt{1+\mathcal{L}_{1}^{2}+1}}$,

(C3): $0<a_{2}<\beta_{n}<b_{2}<\gamma_{n}<c_{2}<\frac{1}{\sqrt{1+\mathcal{L}_{2}^{2}}+1}$,

(C4): $0<\delta<\frac{1}{\|\mathcal{A}\|^{2}}$ and $\sigma>\gamma \rho$.

Then the sequence $\left\{x_{n}\right\}$ generated by algorithm (4.4) converges strongly to $u \in \Gamma_{2}$ (the set of the solutions of (1.9)) provided $\Gamma_{2} \neq \emptyset$.

Algorithm 4.9 For given $x_{0} \in \mathcal{H}_{1}$ arbitrarily, define a sequence $\left\{x_{n}\right\}$ iteratively by

$$
\left\{\begin{array}{l}
v_{n}=\left(1-\zeta_{n}\right) \mathcal{A} x_{n}+\zeta_{n} \mathcal{S}\left(\left(1-\eta_{n}\right) \mathcal{A} x_{n}+\eta_{n} \mathcal{S} \mathcal{A} x_{n}\right) \\
y_{n}=\left(1-\alpha_{n}\right)\left(x_{n}-\delta \mathcal{A}^{*}\left(\mathcal{A} x_{n}-v_{n}\right)\right) \\
x_{n+1}=\left(1-\beta_{n}\right) y_{n}+\beta_{n} \mathcal{T}\left(\left(1-\gamma_{n}\right) y_{n}+\gamma_{n} \mathcal{T} y_{n}\right)
\end{array}\right.
$$

for all $n \in \mathbb{N}$.

Corollary 4.10 Suppose that $\mathcal{T}-\mathcal{I}$ and $\mathcal{S}-\mathcal{I}$ are demiclosed at 0 . Assume that the following conditions are satisfied:

(C1): $\lim _{n \rightarrow \infty} \alpha_{n}=0$ and $\sum_{n=1}^{\infty} \alpha_{n}=\infty$,

(C2): $0<a_{1}<\zeta_{n}<b_{1}<\eta_{n}<c_{1}<\frac{1}{\sqrt{1+\mathcal{L}_{1}^{2}+1}}$,

(C3): $0<a_{2}<\beta_{n}<b_{2}<\gamma_{n}<c_{2}<\frac{1}{\sqrt{1+\mathcal{L}_{2}^{2}+1}}$,

$(\mathrm{C} 4)^{\prime}: 0<\delta<\frac{1}{\|\mathcal{A}\|^{2}}$.

Then the sequence $\left\{x_{n}\right\}$ generated by algorithm (4.5) converges strongly to the minimum norm solution $u^{*} \in \Gamma_{2}$ provided $\Gamma_{2} \neq \emptyset$.

\section{Competing interests}

The authors declare that they have no competing interests.

\section{Authors' contributions}

All authors read and approved the final manuscript.

\section{Author details}

${ }^{1}$ Department of Mathematics, Statistics and Physics, College of Arts and Science, Qatar University, P.O. Box 2713, Doha, Qatar. ${ }^{2}$ Department of Information Management, Cheng Shiu University, Kaohsiung, 833, Taiwan. ${ }^{3}$ Center for General Education, Kaohsiung Medical University, Kaohsiung, 807, Taiwan. ${ }^{4}$ Department of Mathematics, Tianjin Polytechnic University, Tianjin, 300387, China. 


\section{Acknowledgements}

Yeong-Cheng Liou was supported in part by MOST 101-2628-E-230-001-MY3 and MOST 101-2622-E-230-005-CC3. Abdelouahed Hamdi would like to thank Qatar University for providing excellent research facilities under Grant: QUUG-CAS-DMSP-14/15-4

Received: 4 August 2015 Accepted: 15 October 2015 Published online: 08 December 2015

\section{References}

1. Censor, Y, Elfving, T: A multiprojection algorithm using Bregman projections in a product space. Numer. Algorithms 8 221-239 (1994)

2. Xu, HK: Iterative methods for the split feasibility problem in infinite-dimensional Hilbert spaces. Inverse Probl. 26, $105018(2010)$

3. Xu, HK: Averaged mappings and the gradient-projection algorithm. J. Optim. Theory Appl. 150, 360-378 (2011)

4. Byrne, C: Iterative oblique projection onto convex subsets and the split feasibility problem. Inverse Probl. 18, 441-453 (2002)

5. Byrne, C: A unified treatment of some iterative algorithms in signal processing and image reconstruction. Inverse Probl. 20, 103-120 (2004)

6. Wang, F, Xu, HK: Approximating curve and strong convergence of the $\mathrm{CQ}$ algorithm for the split feasibility problem. J. Inequal. Appl. 2010, Article ID 102085 (2010)

7. Dang, Y, Gao, Y: The strong convergence of a KM-CQ-like algorithm for a split feasibility problem. Inverse Probl. 27, $015007(2011)$

8. Yang, Q: The relaxed CQ algorithm for solving the split feasibility problem. Inverse Probl. 20, 1261-1266 (2004)

9. Wang, Z, Yang, Q, Yang, Y: The relaxed inexact projection methods for the split feasibility problem. Appl. Math. Comput. 217, 5347-5359 (2011)

10. Yao, Y, Wu, J, Liou, YC: Regularized methods for the split feasibility problem. Abstr. Appl. Anal. 2012, Article ID 140679 (2012)

11. Yang, Q: On variable-step relaxed projection algorithm for variational inequalities. J. Math. Anal. Appl. 302, 166-179 (2005)

12. Lopez, G, Martin-Marquez, V, Wang, F, Xu, HK: Solving the split feasibility problem without prior knowledge of matrix norms. Inverse Probl. 28, 085004 (2012)

13. Yao, Y, Postolache, M, Liou, YC: Strong convergence of a self-adaptive method for the split feasibility problem. Fixed Point Theory Appl. 2013, Article ID 201 (2013)

14. Censor, Y, Segal, A: The split common fixed point problem for directed operators. J. Convex Anal. 16, 587-600 (2009)

15. Moudafi, A: The split common fixed-point problem for demicontractive mappings. Inverse Probl. 26, 055007 (2010)

16. Moudafi, A: A note on the split common fixed-point problem for quasi-nonexpansive operators. Nonlinear Anal. 74, 4083-4087 (2011)

17. Kraikaew, R, Saejung, S: On split common fixed point problems. J. Math. Anal. Appl. 415, 513-524 (2014)

18. Cui, H, Wang, F: Iterative methods for the split common fixed point problem in Hilbert spaces. Fixed Point Theory Appl. 2014, Article ID 78 (2014)

19. Yao, Y, Liou, YC, Yao, JC: Split common fixed point problem for two quasi-pseudo-contractive operators and its algorithm construction. Fixed Point Theory Appl. 2015, Article ID 127 (2015)

20. Xu, HK: Iterative algorithms for nonlinear operators. J. Lond. Math. Soc. 66, 240-256 (2002)

21. Mainge, PE: Approximation methods for common fixed points of nonexpansive mappings in Hilbert spaces. J. Math. Anal. Appl. 325, 469-479 (2007)

\section{Submit your manuscript to a SpringerOpen ${ }^{\circ}$ journal and benefit from:}

- Convenient online submission

Rigorous peer review

- Immediate publication on acceptance

Open access: articles freely available online

- High visibility within the field

- Retaining the copyright to your article 